Archives

$8 \mid 1991$

Varia

\title{
Retour sur une enquête
}

\section{Mona Ozouf et Jacques Ozouf}

\section{(2) OpenEdition \\ Journals}

\section{Édition électronique}

URL : http://journals.openedition.org/ccrh/2817

DOI : $10.4000 /$ ccrh. 2817

ISSN : $1760-7906$

\section{Éditeur}

Centre de recherches historiques - EHESS

\section{Édition imprimée}

Date de publication : 15 octobre 1991

ISSN : 0990-9141

Référence électronique

Mona Ozouf et Jacques Ozouf, "Retour sur une enquête », Les Cahiers du Centre de Recherches Historiques [En ligne], 8| 1991, mis en ligne le 18 mars 2009, consulté le 19 avril 2019. URL : http:// journals.openedition.org/ccrh/2817; DOI : 10.4000/ccrh.2817

Ce document a été généré automatiquement le 19 avril 2019

Article L.111-1 du Code de la propriété intellectuelle. 


\title{
Retour sur une enquête
}

\author{
Mona Ozouf et Jacques Ozouf
}

1 Cette enquête, entreprise dans les années soixante auprès de 20.000 instituteurs ayant enseigné avant 1914 et alors survivants, avait valu à Jacques Ozouf plus de 4.000 réponses. Il en avait donné un aperçu dans Nous les maîtres d'école, en privilégiant naturellement, pour cette présentation destinée au grand public, les «beaux » témoignages, et, avec le projet, malheureusement contrarié, d'une utilisation ultérieure moins impressionniste. C'est une chance d'avoir pu, après tant d'années, reprendre ce travail, grâce au Centre de Recherches Historiques, au soutien de Louis Bergeron, au concours de plusieurs chercheurs de l'École des Hautes Études ou de l'Institut d'Études Politiques, Jean Prévot, Véronique Aubert, Claire Bernard, et à la bonne grâce de Catherine Gréard, à qui nous n'avons cessé, en cours d'enquête, de demander de nouveaux calculs et d'opérer les ajustements que nous suggéraient les premiers résultats.

2 Pionnière en 1962, et du reste diversement accueillie - Pierre Renouvin avait manifesté son intérêt mais Ernest Labrousse son scepticisme -, l'enquête dont il s'agit porte bien sa date, inspirée qu'elle avait été par une préoccupation à la fois démocratique et ethnologique. Démocratique, puisque l'histoire contemporaine, qui renseigne de façon exubérante sur les votes, discours, débats, commentaires de la classe politique et des militants, est en revanche fort avare de renseignements sur les masses silencieuses et il s'agissait alors, souci devenu depuis un lieu commun, de faire parler ceux qui ne parlent pas. Ethnologique, d'autre part, puisque l'enquête était animée par l'urgence de faire surgir ce qui, faute d'avoir été suscité et consigné, allait s'engloutir.

3 Les limites de cette enquête sautent aux yeux aujourd'hui. Limites du questionnaire ${ }^{1}$ qui était fort long et détaillé, mais dont la raideur laissait peu de chances à une réponse libre et inspirée, et dont le découpage reflétait surtout les curiosités de l'enquêteur : ainsi yavait-il abondance de questions politiques, mais indigence de questions portant sur la pédagogie ou l'exercice du métier, celles-ci étant pourtant liées à celles-là. Par ailleurs, on fournissait aux correspondants une liste des grands événements de la III ${ }^{\mathrm{e}}$ République, de la Commune de Paris à la Revanche, sur lesquels ils étaient invités à réagir. Mais on n'avait pas su imaginer la question qui aurait suggéré aux instituteurs de définir eux- 
mêmes ce qu'était un grand événement et d'apprécier celui qui avait été pour eux décisif. Limites, aussi, dues à la perspective positiviste de l'entreprise : la grande préoccupation de son auteur avait été de construire un échantillon représentatif, et de vérifier, sur tel ou tel point épineux - l'engagement syndical, par exemple, ou l'entrée dans la francmaçonnerie - si les témoignages recueillis correspondaient bien aux données factuelles. Il faut dire que les témoins, tout prêts à capter cette inspiration, s'étaient eux-mêmes évertués à contrôler leur subjectivité et à fournir des récits scrupuleusement vérifiés : la précision de leurs réponses est parfois chronologique (la vérification des grandes dates de leur existence les avait beaucoup occupés), et parfois topograhique (le questionnaire les avait poussés à revisiter les lieux de leur enfance et leurs maisons d'école), elles sont toujours appuyées sur les documents qu'en archivistes minutieux de leurs vies ils avaient conservés. A leurs réponses, ils ont donc ajouté leurs livres de comptes, leurs cahiers de préparation de classe, leurs cartes de sociétaires de la section syndicale. Mais aussi les circulaires imprimées que les directeurs devaient remplir sur le comportement de leurs adjoints, les lettres d'inspecteurs primaires accordant ou refusant un congé, les rapports d'inspection, les discours de distribution des prix. Ils ont retrouvé, qui la correspondance de leur père avec un curé du Second Empire, qui les notes d'un grand-père pris dans la tourmente de 1848. Certains se sont appliqués à dresser la généalogie d'une famille fertile en enseignants, d'autres à dessiner le profil de carrière de tous les normaliens de leur promotion. Ils envoient les photographies des bataillons scolaires, les médailles des sociétés d'agriculture, les poèmes composés pour les banquets de l'Amicale. Enfin, conscients d'avoir, après deux guerres mondiales, abordé aux rives "d'une autre planète ", comme ils disent, et d'avoir vécu ce qu'on ne verra plus, ils ont ajouté des informations ethnographiques. Description des pratiques alimentaires au village, récit des conversations chez le maréchal-ferrant ou au moulin à huile, fête des vendanges, rites du carnaval : tout un monde perdu qu'ils s'ingénient à faire revivre. Bref, ils ont apporté des réponses à des questions qui ne leur avaient pas été posées et considérablement enrichi l'enquête.

D'autre part, même si l'enquêteur n'avait pas su les encourager à la digression personnelle, eux en avaient souvent éprouvé le besoin. Certains d'entre eux, moins obsédés par les contraintes du questionnaire, avaient compris l'invitation qu'il recélait à l'association libre, tenté de retrouver, en arrière de leur point d'arrivée, la ligne sinueuse d'un itinéraire personnel, et s'étaient évadés du cadre fixé. Cette évasion est d'abord matérielle, chez tous ceux qui ont abandonné les tristes feuilles du questionnaire imprimé pour recourir à ce cahier tout neuf dont la blancheur communique la griserie légère de la table rase, et inspire le désir de tout ressaisir par le commencement. Mais intellectuelle aussi bien: ils bousculent l'ordre des questions, préférant la liberté des questions ouvertes à la nécessité des questions fermées. Elle est existentielle enfin: parfois avec élan, parfois avec remords, ils trouent leur exposé d'adresses à leur lecteur, brèves explosions de spontanéité, confidences et aveux, à la fois contrits et heureux. Il est vrai qu'à un questionnaire qui commençait par : «Où êtes-vous né ?", il était tentant de répondre à la manière de Jean-Jacques : "Je suis né à Genève en 1712 d'Isaac Rousseau citoyen et de Suzanne Bernard citoyenne ». La confession est presque immédiatement appelée par ces lignes, desquelles, semble-t-il, doit couler le fleuve de l'existence. «Je suis né à l'écluse $\mathrm{n}^{\circ} 23$ du canal des Ardennes, près du village de Semuy ». "Je suis né dans le Cabardès, où poussent la fougère, l'yeuse et la bruyère ». Ils ont du reste eu eux-mêmes le sentiment de céder à la tentation autobiographique, au point parfois d'intituler leurs réponses "mémoires» ou «mes Souvenirs», et ne reculent pas devant l'odeur de 
religiosité qui flotte autour du mot de «confession». Il n'y manque pas même la sensation de délivrance que promet la bonne confession. Comment conclure, se demande, élève appliqué jusqu'au bout, un de nos témoins? «J'ai l'impression que je viens de faire mon examen de conscience et je suis comme soulagé d'avoir exposé avec franchise mes états d'âme, mes difficultés, mes joies, mes peines ».

5 L'enquête, pour eux, a donc agi comme un révélateur. Pour leur lecteur aussi bien, qui se retrouve après avoir traversé leurs textes, avec des richesses plein les bras. Richesses ambiguës. Car tantôt, pénétrés de « l'utilité » de l'entreprise, ils y répondent avec le souci de faire le portrait - ou de dresser la statue - de l'instituteur français au tournant du siècle : perspective objective où les habite surtout le doute de pouvoir vraiment apporter leur pierre à une œuvre quasi-collective, et l'anxiété de savoir s'ils peuvent prétendre personnellement à la dignité du type. Et tantôt ils se laissent emporter par la nature même de l'exercice à la récapitulation autobiographique : perspective subjective celle-ci, qui à la fois les gêne et les enchante. D'où la tension entre deux exigences disparates, perceptible dans des réponses très riches, qui hésitent entre singularité et généralité.

$C^{\prime}$ est cette surabondance de renseignements qui nous a dicté le traitement du corpus, pour lequel nous avons réalisé deux codages parallèles. Tout d'abord les 4.000 réponses ont été systématiquement codées pour douze variables sociologiques simples (sexe, âge, origine sociale ou géographique, cursus scolaire). Ce qui permet, par comparaison avec la morphologie du corps à l'époque considérée - en gros, le tournant du siècle - d'apprécier les biais de représentativité inhérents au sondage rétrospectif. Nous savons désormais, grâce à ce codage, que notre enquête donne une image globalement assez fidèle du corps des instituteurs. Sur les origines socioprofessionnelles, les sympathies partisanes, l'appartenance aux amicales, nous savons que notre population est conforme à la population d'origine. Nous pouvons aussi mieux mesurer les écarts. Il y a par exemple relativement plus d'hommes dans notre population qu'il n'y en avait à l'origine dans un corps qui était déjà au début du siècle à légère majorité féminine (même si dans l'imaginaire national il restait masculin). A l'évidence, les hommes prennent plus volontiers la décision de répondre au questionnaire et il arrive même que pour les couples d'instituteurs, le mari rédige les deux réponses, ou une réponse unique pour les deux, avec des commentaires ingénus : " A partir de son mariage, la vie de madame X se confond absolument avec la mienne ». Chez nos témoins d'autre part, il y a relativement plus d'instituteurs issus des Écoles Normales que dans le corps de 1900 : tout nous donne à croire que les instituteurs qui ont répondu à l'enquête sont les plus brillants, en tout cas ceux qui ont fait les carrières les plus conformes, et probablement aussi les plus heureuses: avoir trouvé l'élan suffisant pour consigner le bilan d'une vie suppose que celui-ci n'ait pas paru trop décourageant. Notre population est une population satisfaite du parcours opéré, et nos comptages nous permettent de mesurer cette satisfaction. Ils nous donnent aussi la chance de mieux mettre en perspective le témoignage exceptionnel, jadis retenu pour sa richesse d'information ou sa qualité littéraire, mais qui prend désormais tout son relief de se détacher sur la normalité. Bref, cette première investigation a apaisé bon nombre de nos scrupules.

7 Toutefois, devant la difficulté de faire tenir dans ces calculs rustiques des textes très élaborés, très infidèles au déroulement du questionnaire, et le débordant de toutes parts, nous avons tenté un autre codage, extrêmement détaillé celui-ci, en retenant 144 indicateurs, et en débordant nous-mêmes les questions initiales de l'enquête. En voici deux exemples. L'une des questions était : «Aviez-vous une admiration pour un homme 
politique, et lequel?», ce qui permet de reconstituer le Panthéon explicite de nos instituteurs. Mais parallèlement, en comptabilisant toutes les mentions révérentes spontanément venues sous la plume de nos correspondants, nous avons pu dessiner un second Panthéon, à un niveau un peu moins réfléchi et moins élaboré. Autre exemple : avec la mention de la profession des grands-parents, des parents, des enfants et parfois des petits-enfants, nous pouvons saisir objectivement le profil de la lignée (ascendante, descendante, immobile). Mais à partir des commentaires, satisfaits ou insatisfaits, qui accompagnent ces renseignements, il nous est permis d'en saisir parallèlement la représentation subjective.

Ce travail, très poussé et très dévoreur de temps, nous l'avons pratiqué sur la moitié seulement de notre enquête, en vérifiant naturellement la correspondance de la seconde population à la première. Ce qui apporte, bien au-delà des données factuelles, une masse d'informations sur l'image du métier, l'univers de valeurs et de normes de nos instituteurs, les systèmes idéologiques. Une fois croisées entre elles, elles nous renseignent sur la part respective tenue par la famille et par l'école dans le choix du métier, sur le rôle que joue dans ce choix le désir, la contrainte, ou plus prosaïquement le rang dans la fratrie, sur la profession du conjoint en relation avec le type d'études de l'enquêté, sur l'intérêt politique ou l'appartenance partisane, dans leurs rapports avec l'âge, le sexe, la filière professionnelle, l'origine géographique, les croyances religieuses, le degré de la pratique, etc. Donc, une cascade de résultats enfin chiffrés.

Qu'ont-ils, exactement, à nous apprendre ? Il nous semble d'abord qu'ils sont de nature à nuancer la représentation traditionnelle des instituteurs comme ce corps homogène dont les métaphores (celles de l'armée, les «hussards» ou les «fantassins », ou celles de l'église, le "sacerdoce», ou "les saints sans espérance ») supposent un engagement explicite, massif, sans retour. Sans doute pourront-ils répondre à des interrogations redevenues aujourd'hui très présentes dans notre vie publique. Au moment où la déroute des idéologies invite tant de nos contemporains à redessiner dans le passé la figure exemplaire du républicanisme de la Troisième et à réinstaller sur son socle la statue de Jules Ferry, nos instituteurs nous aident à mieux comprendre le contenu de l'idée républicaine, dans ses rapports avec la tradition révolutionnaire, avec l'idée chrétienne, avec l'idée socialiste.

10 Il est impossible de faire tenir ici ces enseignements, qu'on trouvera bientôt dans un livre qui doit être publié dans la collection Gallimard-le Seuil-Hautes-Études. Nous nous contenterons d'évoquer quelques-uns des étonnements que nous a réservés notre travail et quelques-unes des hypothèses où ils nous engagent. Nous avons par exemple été surpris du nombre élevé de non-réponses chez nos témoins lorsqu'il s'agissait de la fondation de l'école laïque. Certes, il s'agit d'un événement qu'ils n'ont pas vécu, mais qui n'est pas pour eux, au moment qu'ils sont censés ressaisir, plus éloigné dans le temps que ne l'est pour nous la crise de 1968, et qui est lié de façon beaucoup plus journalière et plus intime à leurs existences. Détachement énigmatique, par conséquent, et que confirmerait aussi bien la place modeste qu'ils ont réservée à Jules Ferry dans le Panthéon de leurs grands hommes : $3 \%$, alors que $33 \%$ citent Jaurès, et l'homme des lois laïques vient seulement au septième rang de leurs admirations, dans une liste qui après Jaurès compte Briand, Herriot, Clemenceau, Poincaré. Il y a là une anomalie à comprendre, mieux mise en valeur encore par cette constatation: le thème de la lutte anticléricale les trouve en revanche très vivement alertés et suscite chez eux une passion égale à celle que l'Affaire Dreyfus soulève. La laïcité n'a donc pas cessé d'être un combat journalier sur le terrain, 
mais curieusement il est décroché d'une histoire qui paraît s'être éloignée dans un temps très reculé, dans un "ancien temps" que, quelle que soit l'âpreté des querelles villageoises, on ne croit pas possible de voir revenir, qu'on étend en arrière abusivement et dont on retrace les luttes sur un mode héroïsé. Le passé de l'école laïque est pour eux le « il était une fois » du conte, récité, célébré, vénéré, mais tenu pour décidément révolu.

Illustration de ce décalage, source nouvelle d'étonnements au regard de l'intégrisme laïc postulé dans le portrait collectif des instituteurs, l'affaire des "emblèmes ", resurgie aujourd'hui dans notre mémoire collective à travers l'affaire des foulards. Des circulaires de 1882, relancées par le combisme, stipulaient que les emblèmes religieux, crucifix, images, statues, ne devaient pas figurer dans les locaux scolaires : il s'agissait d'étendre le droit commun au seul des services publics qui ait conservé l'attache confessionnelle. L'extrême diversité des situations locales évoquées par nos témoins, la conscience aigüe de cette bigarrure, font comprendre des faits qui sans elles paraîtraient énigmatiques : la fréquence des compromis sur le terrain, l'extrême lenteur avec laquelle les mesures de laïcisation sont mises en place, le caractère précautionneux des parades imaginées, l'indifférence enfin aux grandes dates nationales qui scandent le conflit entre l'Église et l'État. On peut la mesurer au traitement assez négligent que ces instituteurs réservent à la loi de séparation. Sans doute trouve-t-on quelques récits flamboyants des inventaires et de la place qu'ils y ont prise, soit comme enfants et adolescents qui ce jour de grand spectacle font l'école buissonnière, soit comme adultes, soldats désignés pour protéger les fonctionnaires, ou instituteurs secrétaires de mairie, un peu confus de se trouver pour cette besogne aux côtés du maire et du préfet. Mais le fait de la séparation lui-même, dont ils approuvent les raisons profondes (même si certains, de l'observatoire des années soixante, voient le bénéfice que l'Église en a finalement tiré et la liberté qu'elle y a puisée de jeter l'anathème sur l'école publique), leur parait un événement naturel, qui du reste intervient un quart de siècle après les lois laïques, un accomplissement dépourvu de toute surprise, imposé par un réflexe de défense. Un bon test de cette indifférence est dans nos réponses l'absence de toute réaction à la discussion qui oppose certains radicaux - on sait qu'ils ont combattu la loi pour préserver le contrôle de l'État sur l'action temporelle des Églises et lui conserver un rôle efficace de police des cultes - et les socialistes qui soutiennent les dispositions d'inspiration libérale. Dans l'ensemble, les instituteurs approuvent le " geste » qu'a eu le gouvernement de laisser les églises à la disposition des fidèles et du clergé, mais le débat n'a pas marqué leurs mémoires, alors qu'ils ont gardé le vif souvenir de l'affrontement vécu dans un espace plus modeste, et interprété par des acteurs identifiables. Et c'est aussi l'existence et la proximité du combat quotidien qui empêchent ces sympathisants du socialisme de partager et même d'apercevoir la critique socialiste à l'égard du laïcisme. Sans doute certains d'entre eux sont-ils assez lucides pour déceler dans cette guérilla quotidienne les risques de sectarisme et parfois les décrire. Mais il ne leur vient pas à l'esprit que l'anticléricalisme bourgeois puisse s'entendre à faire manger du prêtre pour retenir les démunis de manger du patron. L'hypothèse d'un anticléricalisme de diversion, émise par Guesde, développée par Lafargue dans les journaux que pourtant ils lisent, ne semble pas les effleurer. C'est probablement que pour parvenir à concevoir cet anticléricalisme en idée, il faudrait n'avoir pas à rencontrer chaque jour les conflits suscités au village par le cléricalisme en action.

12 Avec leurs réactions aux grands événements de la vie nationale comme aux menus incidents de la vie locale, avec les listes de livres qu'ils lisaient, des journaux qu'ils achetaient, des revues pédagogiques auxquelles ils étaient abonnés, le nom des 
éditorialistes qu'ils préféraient, avec les commentaires dont ils assortissent ces bilans, nous pouvons donc dessiner avec précision la carte de leur culture politique. Une culture pourvue d'une exceptionnelle cohérence, puisque toutes les influences se prêtent main forte et se redoublent: les livres sont désignés par les parents et par les maîtres, les grands événements sont interprétés par l'école, les revues pédagogiques elles-mêmes accueillent le commentaire de l'actualité et développent une morale de la politique, toute mêlée à la politique de la morale. Tout cela constitue une tradition particulièrement robuste. Et pourtant, quand on l'interroge, on est frappé de son caractère pelliculaire : dix-septième et dix-huitième siècles presque absents des lectures, rareté des événements du passé à paraître dignes du regard rétrospectif, pauvreté des allusions à la Révolution française, fait surprenant lorsqu'on songe que de ces hommes Jules Ferry avait explicitement voulu faire des « fils de 89 » (un seul sur nos quatre mille témoins, dont des historiens sérieux continuent à écrire qu'ils enseignaient la religion robespierriste a pour Robespierre une mention révérente). Bref, leur univers de références est presque tout entier délié du passé. Ils dévalorisent le surplomb des traditions, assimilent plus ou moins confusément à la servitude toutes les relations verticales de dépendance et parfois même d'admiration, et célèbrent l'horizontalité démocratique. De cette volonté d'autonomie, notre enquête comporte maints exemples: de la Revanche qu'ils refusent de se laisser dicter par leurs aînés (exemple particulièrement éloquent puisque qui dit Revanche dit accomplissement par la génération nouvelle de ce qui est désigné par la génération précédente) à l'Affaire Dreyfus qui met à mal la tradition de l'obéissance militaire, jusqu'au syndicalisme balbutiant qui naît de l'affrontement entre les jeunes adjoints et les vieux directeurs. Il a là quelque chose à comprendre, une relation très particulière au passé, qui est vénéré mais pas contraignant, qui a force d'illustration mais pas force de loi, et où la seule tradition qui fasse autorité est la capacité des hommes à secouer l'autorité de la tradition. Comprendre l'originalité de cette culture politique et par là l'originalité du républicanisme français nous paraît aujourd'hui, par delà les résultats d'histoire sociale qu'elle visait initialement et qu'elle apportera, l'intérêt majeur de cette enquête et la promesse de son rajeunissement.

\section{Questionnaire}

13 - Quel est votre nom? Votre prénom?

14 - Quelle est votre date de naissance?

15 - A quelle date êtes-vous entré dans l'Enseignement Primaire?

\section{Origines sociales.}

16 - Où êtes-vous né ? Hameau, village, ville?

17 - Où avez-vous été élevé ? (id.)

18 - Quelles sont vos origines sociales (profession de vos parents, niveau de fortune de ceuxci, composition de cette fortune: par exemple avaient-ils des revenus mobiliers, des propriétés...; niveau intellectuel de vos parents ?...) ? 


\section{Carrière.}

19 A) Pourquoi êtes-vous devenu instituteur (vocation pédagogique; influence de votre famille ou de votre entourage ; nécessité matérielle ; autres causes) ?

B) Dans quelles conditions avez-vous fait vos études pour devenir instituteur? Où? Avec ou sans aide matérielle de votre famille? en aidant votre famille?

c) Quelle a été votre carrière (dates extrêmes; passage à l'École Normale ; principaux postes)?

D) Avez-vous exercé, avant ou après l'enseignement primaire une autre profession ?

E) votre carrière a-t-elle été marquée par des incidents quelconques?

- avec vos supérieurs hiérarchiques (directeur, inspecteur)?

- avec d'autres rouages de l'administration?

- avec la population (municipalités, notables locaux, autorités ecclésiastiques) ? Lors des laïcisations par exemple?

Si oui, lesquels ? Et à quoi les attribuez-vous?

\section{Conditions de vie.}

A) Vous êtes-vous marié ? Quand ? Avez-vous eu des enfants ? Combien?

B) Vous souvenez-vous de votre traitement de début? De votre traitement en 1914 ? Estimiez-vous ce traitement suffisant?

Aviez-vous d'autres ressources (autre activité professionnelle, revenus de propriétés...) ? Votre conjoint était-il instituteur? Exerçait-il une autre profession (au moment de votre mariage ; ensuite) ? Si oui, laquelle? Avait-il d'autres revenus (rentes, propriétés)?

Quel jugement aviez-vous à l'égard du niveau de vie que vous procurait l'ensemble de vos revenus familiaux?

C) Quelles étaient, pendant votre carrière, vos relations sociales ? Avec des collègues, avec d'autres couches de la population?

Que pensait-on des instituteurs dans les milieux qui vous entouraient?

Quelle importance accordiez-vous à votre métier dans l'ensemble de votre vie? Aviezvous d'autres activités (liées à l'école ou non)? A quoi, de préférence, occupiez-vous vos loisirs?

E) Si vous avez eu des enfants, désiriez vous le voir embrasser la carrière d'instituteur? Ou une autre carrière enseignante. Les y avez-vous poussés? Ou bien désiriez-vous les orienter vers une autre profession?

Qu'ont-ils choisi finalement?

\section{Lectures.}

A) Aviez-vous des lectures favorites, parmi les auteurs contemporains, parmi les écrivains plus anciens ? Ont-elles exercé une influence sur votre pensée?

B) Lisiez-vous avant 1914 des journaux et des revues (politiques, littéraires,...) ? Si oui, lequel ou lesquels? 
C) Étiez-vous abonné à une des revues pédagogiques (Manuel général, Le Volume, Le Journal des Instituteurs, l'École Nouvelle, La Revue de l'Enseignement Primaire, L'École laïque)?

Si oui, à laquelle et pouvez-vous dire pourquoi ?

\section{Le syndicalisme et les problèmes sociaux}

31 A) Avez-vous été membre d'une Amicale d'Instituteurs ou d'Institutrices? Quand et où? Y avez-vous joué un rôle dirigeant (président, secrétaire,...) ?

Étiez-vous favorable ou hostile à l'évolution des Amicales vers le syndicalisme?

B) Avez-vous été membre d'un syndicat d'instituteurs avant 1914 ? Quand? Aviez-vous une responsabilité dans le syndicat?

C) Que pensiez-vous du syndicalisme ouvrier, du syndicalisme enseignant, et de leurs liens éventuels?

D) Comment avez-vous réagi devant les transformations sociales ? Par exemple l'accès des femmes à des métiers nouveaux.

\section{Les questions politiques et philosophiques}

C) Comment avez-vous réagi devant les grands événements politiques de la IIIe République, ceux dont vous avez été témoin ou ceux qui étaient encore très récents? Par exemple : La Commune de Paris ; le Seize Mai 1877 ; l'Affaire Dreyfus ; la fondation de l'école laïque ; la Revanche (connaissiez-vous la "Ligue des Instituteurs patriotes" ?) ; la lutte anticléricale et la Séparation; la politique coloniale; le socialisme et l'internationalisme; les rivalités internationales et le déclenchement de la guerre de 1914-18 (en particulier l'institution du service de 3 ans).

D) Avez-vous eu des options politiques précises avant 1914 ? Avez-vous été membre d'un parti politique ? Avez-vous été sympathisant d'un parti ou d'une tendance politique?

Pouvez-vous préciser vos options politiques à propos de consultations électorales par exemple (élections générales de mai 1914 par exemple) ? Pouvez-vous en expliquer les raisons?

Que pensiez-vous en particulier du socialisme?

Aviez-vous une admiration pour un homme politique ? Pourquoi ?

E) S'il y avait eu un recensement sur les idées philosophiques de la population, qu'auriezvous répondu? Que vous étiez catholique (pratiquant ou non), protestant (id), librepenseur?

Vous êtes-vous marié religieusement? Avez-vous fait baptiser vos enfants?

Connaissiez-vous la franc-maçonnerie? Qu'en pensiez-vous? Avez-vous eu des liens avec elle? Avez-vous connu des francs-maçons?

P.S. - Si vous disposez de documents relatifs aux instituteurs et à l'enseignement primaire de 1871 à 1914, voudriez-vous avoir l'obligeance de me les confier provisoirement afin que je puisse en prendre connaissance (ou bien, s'ils ne vous sont plus utiles, de me les remettre)? 


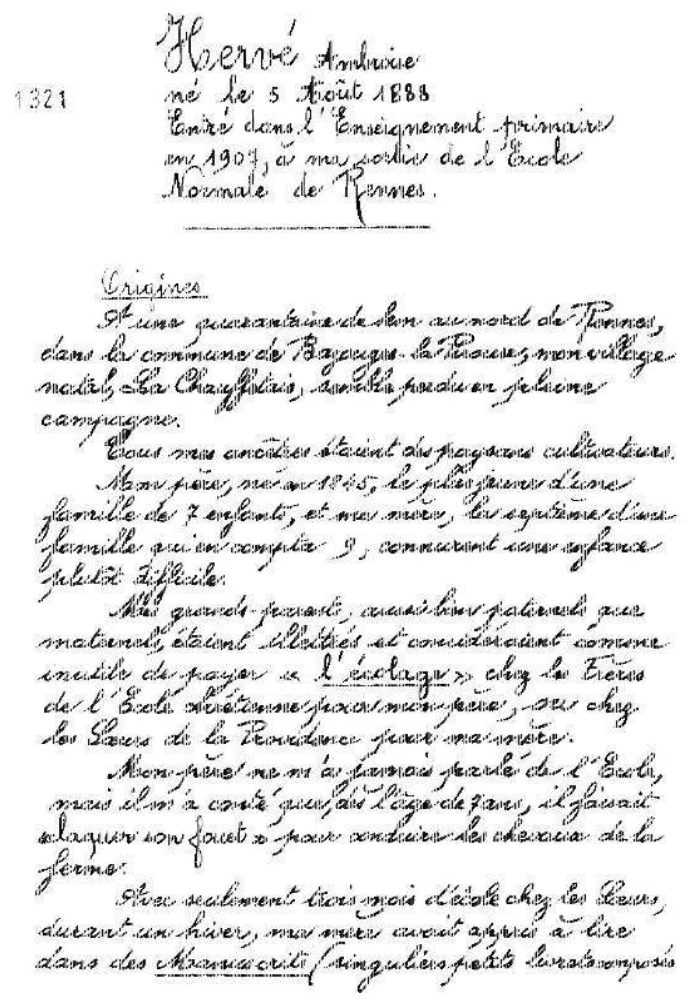

\section{NOTES}

1. Voir en annexe le questionnaire accompagné d'un exemple de réponse.

\section{AUTEURS}

\section{MONA OZOUF}

Mona Ozouf est directeur de recherche au CNRS. Elle est membre du Centre de Recherches Historiques.

\section{JACQUES OZOUF}

Jacques Ozouf est directeur d'études à l'EHESS. Il est membre du Centre de Recherches Historiques. 